E3S Web of Conferences 2, 03008 (2014)

DOI: $10.1051 / \mathrm{e} 3$ sconf/ 20140203008

(C) Owned by the authors, published by EDP Sciences, 2014

\title{
Dalla memoria al paesaggio. Note per un approccio olistico al territorio
}

\section{From memory to landscape. Notes for a holistic approach to the territory}

\author{
Guido Montanari \\ Interunivesity Department of Regional and Urban Policies and Studies, Politecnico di Torino, 10125 Torino, Italy
}

\begin{abstract}
Riassunto. Nelle relazioni e nel dibattito del convegno è stato messo l'accento soprattutto sugli aspetti quantitativi, matematici e "fisici", che evidenziano la contraddizione tra crescita economica e uso incontrollato delle risorse naturali, in un sistema finito come è quello del nostro pianeta. Questo contributo vuole focalizzare l'attenzione su un aspetto che è rimasto ai margini degli interventi: la dimensione della memoria e della tradizione, cioè del rapporto culturale, sociale e psicologico dell'uomo con i luoghi della propria vita, siano essi urbani o rurali. Questi luoghi costituiscono il "paesaggio", come sintesi di valori naturali e culturali. Tuttavia il "diritto al paesaggio" come uno dei diritti fondamentali dei cittadini, sancito dalla Costituzione e dalla legislatura internazionale, resta un concetto poco approfondito che implica invece un nuovo approccio culturale e politico.
\end{abstract}

\begin{abstract}
The talks and the debate of the conference have stressed first of all the quantitative, mathematical and "physical" aspects which highlight the contradiction between economic growth and unrestrained use of natural resources, in a finite system like the one of our planet. The purpose of present contribution is to draw the attention on an aspect remained on the margin of the other talks: the dimension of memory and tradition, i.e. of the cultural, social and psychological link of human beings with the places of their life, be they urban or rural. These places form the "landscape", as a synopsis of natural and cultural values. However the "right to landscape" as one of the fundamental rights of citizens, recognized by the Constitution and by the international legislation, still is a not well scrutinized concept, which rather implies a new cultural and political approach.
\end{abstract}

La lotta per la salvaguardia delle risorse naturali, la svolta verso una ricerca scientifica responsabile e per una tecnologia rispettosa degli uomini e dell'ambiente devono integrare la conoscenza e la salvaguardia dei territori, non soltanto come risorse indispensabili per la vita e per il benessere fisico delle persone, ma anche come "scrigni di memoria", luoghi dove sono raccolte le fonti materiali e culturali "non rinnovabili" che sostanziano le specificità psichiche e morali degli individui e delle comunità, che sono cioè alla base della loro stessa esistenza e della loro coscienza di soggetti portatori di diritti inalienabili.

In ogni organizzazione sociale la possibilità di consegnare ai posteri la riflessione teorica e la dimensione esperienziale sviluppate nel corso del tempo è aspetto determinante di civiltà. La memoria e il racconto del passato costituiscono infatti le basi identitarie di ogni comunità umana e diventano tradizione (dal latino tradere, trasmettere, come ricorda Theodor W. Adorno1) sulla base dei documenti, appunto, tramandati di 
generazione in generazione. Anche se, come dice Fernand Braudel2, lo storico "inventa" le sue fonti per costruire il suo racconto, è purtuttavia il territorio che conserva le testimonianze del passato, collocate sul crinale incerto tra materiale ed immateriale, tra tangibile e intangibile.

La storia si costruisce attraverso una pluralità di fonti, testi e documenti, ma se le testimonianze del passato fossero tutte cancellate potrebbe ancora esistere una storia? Un presente immanente, senza storia, non è forse alla base dell'incubo orwelliano? E non è forse questo uno dei rischi a cui è esposta la nostra società contemporanea dell'effimero, dell'immediato, del precario?

La civiltà stessa è connessa alla nascita della storia e si caratterizza proprio per la volontà di preservare $\mathrm{i}$ documenti del passato come memoria collettiva, dando vita a musei, archivi, biblioteche e monumenti (dal latino monere: ammonire, ma anche ricordo, testimonianza). Infatti se è vero che la storia è scritta dai vincitori, è altrettanto vero che è compito di una società democratica garantire cha la storia sia continuamente approfondita e verificata, dando voce anche ai vinti, agli emarginati, alle minoranze. Per fare questo si deve garantire la conservazione dei luoghi e dei documenti, non soltanto di quelli aulici e riconosciuti come monumenti, ma anche di quelli "minori", magari testimonianza di dolorose vicende della nostra contemporaneità, come è il caso per le tante tragedie del Novecento, l'epoca più violenta della storia dell'umanità3.

Si tratta di un processo non scontato che si basa su una consapevolezza culturale da costruire e sull'approfondimento di procedure e di tecniche non sempre consolidate. Si pensi all'impegno necessario per tramandare materiali fragili come l'intonaco delle celle di una prigione scalfito dai reclusi o quello di un muro bucato dalle pallottole delle fucilazioni. Oppure alla difficile conservazione dei campi di sterminio nazisti o dei villaggi di montagna, teatro della resistenza antifascista e dell'eccidio di civili e di partigiani. Ma si pensi anche agli ostacoli spesso frapposti all'accesso agli archivi di governi, di tribunali e di polizie.

Il territorio antropizzato, con la sua sedimentazione complessa di architetture, di infrastrutture, di insediamenti è il forziere nel quale sono custoditi, anche se talvolta misconosciuti, i tanti e variegati documenti che raccontano le condizioni di vita, il lavoro, le aspirazioni di intere comunità, costituendo i

1

"L'oblio è disumano perché fa dimenticare la sofferenza accumulata: giacché la traccia della storia nelle cose, nelle parole, nei colori e nei suoni è sempre quella della passata sofferenza. Per questo la tradizione si trova oggi davanti a una contraddizione insolubile: nessuna è attuale né da risuscitare, ma quando ogni tradizione è spenta, la marcia verso la disumanità è iniziata”. T. W. Adorno, Parva Aestetica (Frankfurt am Main 1967), Feltrinelli, Milano 1979, p. 35.

2

F. Braudel (a cura di), La storia (Paris 1958), Laterza, Roma-Bari 1974, pp. 153193.

3

E. J. Hobsbawm, Il secolo breve. 1914/1991. L'epoca più violenta della storia dell'umanità (New YorkLondon 1994), BUR, Milano 2000. 
fondamenti di memorie e di saperi collettivi. Sono testimonianze il cui valore simbolico è straordinario, ma la cui consistenza materiale è spesso estremamente fragile4. Da qui il problema della loro individuazione, documentazione e conservazione, a scala di territorio che coinvolge il dibattito disciplinare e le tecniche del restauro, ma anche e soprattutto le politiche di trasformazione territoriale e le normative urbanistiche. Inoltre è necessaria una riflessione specifica quando questa attenzione si rivolge a testimonianze che ben poco hanno dell'appeal estetico proprio dei beni storico-artistici, di riconosciuto valore.

Sono molti gli esempi della difficoltà di questo processo di conoscenza e di conservazione. Per esempio in molti Paesi che si stanno affacciando allo sviluppo si assiste alla distruzione di interi territori e di quartieri storici, come gli hutong di Pechino, per fare spazio alla speculazione edilizia, ad una edilizia quantitativa di bassa qualità, a grandi opere di dubbia utilità, ma decise in nome del preteso "progresso" della globalizzazione. Un analoga miope visione caratterizza la trasformazione dei nostri territori. Preziose testimonianze di attività rurali che potrebbero essere rilanciate costituendo occasioni di sviluppo locale, come colture, canalizzazioni, terrazzamenti sono distrutte da infrastrutture stradali e ferroviarie, da capannoni produttivi, da impianti fotovoltaici a terra, da monocolture estensive, realizzati nella completa indifferenza alle qualità paesaggistiche e ambientali.

Negli ambiti urbani e periurbani si assiste alla distruzione di quartieri operai e di fabbriche, simboli del lavoro e della presa di coscienza di generazioni di lavoratori, quando non del loro sacrificio estremo, come la miniera di carbone di Marcinelle5, oppure le tante "fabbriche della morte", sparse sul nostro territorio6. Nonostante la sempre più diffusa consapevolezza tra tecnici e intellettuali intorno ai valori del patrimonio industriale e malgrado l'impegno di cittadini, di associazioni e talvolta della magistratura, sono rari i progetti di conservazione e di riuso, anzi spesso si assiste alla volontà di oblio e di distruzione da parte di Amministrazioni pubbliche poco attente7: unica "valorizzazione" possibile del territorio sembra essere quella della urbanizzazione e della cementificazione8.

4

G. Montanari, Memoria fragile, lezione aperta, Politecnico di Torino, Salone d'onore del Castello del Valentino, Torino, 27 gennaio 2011.

5

Dove l'8 agosto del 1956 nella miniera di carbone vicino a Charleroi, in Belgio si sviluppa un incendio a 975 metri di profondità nel quale muoiono 262 minatori migranti, di cui 136 italiani. Il cancello di entrata alla miniera e diversi monumenti ricordano sul posto la tragedia.

6

Ricordo i casi della Eternit di Casale Monferrato (fondata nel 1906), la cui proprietà è stata recentemente condannata per la responsabilità della morte e malattia di centinaia di lavoratori. Tra le molte altre che si potrebbero ricordare, sono state oggetto di noti provvedimenti giudiziari l'Ipca di Ciriè, in Provincia di Torino (attiva dal 1922 al 1982), l'Acna di Cengio (Savona) e l'Ilva di Taranto tuttora attiva, tra molte polemiche.

7

- E' il caso dell'Amministrazione di Torino che ha condotto la trasformazione di circa 10 milioni di metri quadrati di aree industriali dismesse con scarsa attenzione alla conservazione del patrimonio storico. Tra gli episodi più 
Mettere in campo pratiche concrete di conservazione implica una nuova ottica con qui guardare al territorio e alla sua pianificazione, con cui avviare studi sulle testimonianze, sviluppare tecniche di individuazione, di documentazione e di restauro che devono coinvolgere le popolazioni locali e i giovani attraverso azioni di conoscenza e di fruizione di luoghi, ambienti e manufatti.

La consapevolezza intorno alla tutela del patrimonio storico, anche se nel nostro Paese ha radici storiche lontane, è una conquista della modernità, sviluppata e radicata in ogni società democratica. Essa si riferisce ai documenti materiali, ma anche alle tecniche, alle culture e non può limitarsi all'istituzione di musei e all'apposizione di "vincoli" ai beni storico artistici, ma piuttosto deve individuare nella riconoscibilità della stratificazione storica dei territori il più ampio concetto di paesaggio come diritto dei cittadini. A questo proposito è doveroso ricordare che la nostra Costituzione, una delle più avanzate del mondo, pone tra i principi fondamentali "la tutela del paesaggio e del patrimonio storico artistico della Nazione" (art. 9)9. E se si pensa agli effetti della tutela del paesaggio sulle condizioni di benessere psicofisico dei cittadini si capisce che questo principio non è una affermazione astratta, ma è strettamente correlato al diritto alla salute (art. 32) e alla limitazione della libertà d'impresa, in funzione del benessere sociale (art. 41).

La consapevolezza dell'importanza della conservazione dei beni culturali e ambientali è sancita a livello internazionale dall'UNESCO con la Convenzione sul patrimonio mondiale del 1972 che promuove la schedatura di siti naturali e antropizzati di straordinario valore culturale e ambientale ritenuti "patrimonio dell'umanità", di cui si propone la conservazione e la valorizzazione10. Questo accordo internazionale è poi integrato dalla Convenzione internazionale sui beni immateriali (2003)11 che, oltre ad allargare il

infelici che hanno suscitato contestazioni, la distruzione di notte, con le ruspe protette da ingenti forze dell'ordine, della storica fabbrica di automobili Diatto, nel quartiere operaio di San Paolo, e la prevista "valorizzazione" immobiliare dell'area della Tyssen-Krupp dove un incidente dovuto a incuria e colpa della direzione ha portato alla morte di otto lavoratori.

8

Nella una vasta documentazione sui danni derivanti dallo spreco del suolo naturale e agricolo, in Italia, mi limito a segnalare S. Settis, Paesaggio costituzione cemento. La battaglia contro il degrado civile, Einaudi, Torino 2010.

9

Costituzione Italiana, 1948, art. 9.

10

Parigi, 21 novembre 1972.

11

Ratificata dall'Italia nel 2007. 
campo della conservazione dei beni materiali a quelli immateriali, integra la tutela delle fonti come strumento per costruire e diffondere la storia delle comunità e della loro memoria.

Sullo sfondo di questi provvedimenti, frutto di un ampio dibattito internazionale, si situa la Convenzione europea del Paesaggio (2000) che offre un utile strumento concettuale e normativo per strappare un termine talvolta ancora interpretato riduttivamente, alla sua usurata accezione estetica. Si legge infatti nella Convenzione:

"Paesaggio designa una parte di territorio nella sua interrelazione di natura e di cultura così come percepita dalle popolazioni il cui carattere risulta dall'azione di fattori naturali elo umani e dalle loro interrelazioni" 12.

Dunque tenendo presenti questi riferimenti di legge e assegnando nuovo riconoscimento alle testimonianze materiali e immateriali del passato, si pone la riflessione e l'azione concreta sui temi della memoria, imperativo democratico sempre più attuale in un momento storico come il presente, nel quale si moltiplicano i deliri negazionisti, i tentativi di appropriarsi della storia per darne una lettura di parte, oppure i segnali preoccupanti di un oblio collettivo e di una "paura della storia"13.

Come insegna una vasta letteratura le pratiche di annichilamento della volontà e della personalità dell'individuo si basano in primo luogo sulla sua spogliazione (fisica e morale), finalizzata alla perdita della propria identità14. La distruzione del passato, la costruzione di quartieri tutti uguali, senza rapporto con il contesto, privi di specificità e di qualità urbana è alla base della trasformazione dei luoghi in “nonluoghi”, come descritto dall'antropologo Marc Augè15. Questo processo, provocando la perdita di radicamento dei cittadini nel proprio territorio e impedendo la possibilità di riconoscere in uno spazio

12

Convenzione Europea del Paesaggio, Firenze, 2000, ratificata dall'Italia con legge 14 del 2006 e dal Codice dei Beni Culturali, 2008.

13

Mi riferisco per esempio al ridimensionamento della storia che emerge nelle recenti "riforme" dei programmi didattici della scuola dell'obbligo. Per un approfondimento: Manifesto dei Cinquecento, Chi ha paura della storia? in http://manifesto500.altervista.org.

14

La pratica del taglio dei capelli e della privazione dei propri vestiti è tra le più frequenti vessazioni dei detenuti utilizzata nei campi di concentramento dei regimi dittatoriali e non del tutto superata anche ora, nel nostro Paese. Punto di partenza per una riflessione su questi temi resta H. Harendt, La banalità del male (New York 1966), Feltrinelli, Milano 2003.

15

M. Augè, Nonluoghi, introduzione a una antropologia della surmodernità (Paris 1992) Eleuthera, Milano 1996. 
determinato gli affetti e le memorie, è tra le cause dei noti fenomeni sociali di emarginazione, di disagio psichico, di "spaesamento", tipici dei quartieri di periferia e dei territori degradati, spesso teatro di violenza e di sommosse.

Al contrario è proprio nella possibilità di riconoscere la sacralità e la specificità del luogo, il genius loci, dell'antichità greca e romana16, che possono e devono essere trovati gli elementi guida per un progetto di trasformazione del territorio e della città, rispettoso della storia e della memoria, portatore di civiltà.

In epoca contemporanea il rapporto degli architetti con il passato è stato interrotto dalla cesura con la tradizione attuata dalle Avanguardie artistiche17, alla ricerca di un'arte e di un'architettura "del proprio tempo". Tuttavia il tentativo di rinnovamento radicale dell'architettura attuato dal Movimento moderno va in crisi nel secondo dopoguerra, sia in conseguenza del disincanto provocato dalle tragedie belliche, sia del bilancio negativo di molte esperienze improntate alla "rottura con la tradizione", ma incapaci di dare risposta ai problemi sociali dell'abitare. Il vasto dibattito che si sviluppa in questo periodo in Europa intorno al "cuore della città", e alla ricostruzione postbellica si articola proprio a partire da una nuova attenzione ai centri urbani e alla loro sedimentazione storica, sociale e culturale.

In questo contesto di ripensamento della modernità Aldo Rossi propone di rapportarsi alla città come ad un insieme inscindibile di architetture tra cui non conta tanto il singolo manufatto, quanto le interrelazioni tra loro e tra loro e il contesto18. Analogamente gli studi sui paesaggi agrari e rurali19, sviluppati a partire dagli anni Sessanta e l'evoluzione del concetto di monumento, da emergenza isolata a tessuto storico complesso, mettono sempre più in rilievo il ruolo del territorio come frutto di stratificazione, ricco di culture specifiche, di tecniche costruttive, di sistemi produttivi, in grado di essere riconosciuti e valorizzati come risorse di civiltà.

Un Paese ad alta densità di beni culturali e di bellezze naturali come il nostro, ricco di una radicata tradizione in merito alla conservazione del patrimonio, manifesta grande difficoltà nel mettere in atto pratiche di conservazione efficaci e condivise. Da un lato un quadro normativo confuso e bizantino, articolato in una inutile conflittualità tra i livelli nazionali e locali della tutela, dall'altro una persistente settorializzazione e non comunicazione tra i saperi che guardano al territorio, divisi tra urbanistica, ecologia

16

F. Bevilacqua, Genius loci. Il dio dei luoghi perduti, Rubettino editore, Soveria Mannelli 2010.

17

M. De Micheli, Le avanguardie artistiche del Novecento (1959), Feltrinelli, Milano 1975.

18

A. Rossi, L'architettura della città (1966), CittàStudiEdizioni, Milano 1995. 
e storia dell'arte, contribuiscono a fare del "Bel Paese" una terra di conquista dove il territorio è visto da molti imprenditori, politici e amministratori come una preda da cui trarre profitti immediati, nell'indifferenza ai valori della memoria e della sua trasmissione. Le sempre più frequenti tragedie legate al dissesto idrogeologico, l'impressionante consumo di suolo, la scarsa qualità urbana dei nuovi insediamenti, sono alcuni degli aspetti più noti di questa incapacità di elaborare una visione più ampia e una gestione oculata del paesaggio20.

Elementi di resistenza e di inversione di rotta possono essere trovati nelle prese di posizione di associazioni ambientaliste e culturali, nelle azioni di informazione, di resistenza e di contrasto messe in campo dai tanti comitati di cittadini che si organizzano per difendere e riqualificare i loro contesti21. Ma non basta: l'università e la ricerca devono sviluppare le interazioni tra le varie discipline e approfondire gli aspetti scientifici che riguardano la conservazione e la riqualificazione del paesaggio. Per fare questo è necessario un nuovo approccio olistico che, oltre a tenere in conto gli aspetti economici e fisici del territorio, assegni nuova attenzione alla memoria e alla riconoscibilità da parte dei cittadini delle qualità sedimentate e delle vocazioni dei luoghi della loro vita. In questo impegno di conoscenza e di proposta il Politecnico di Torino, anche in virtù della sua lunga tradizione di studi sul tema dei beni architettonici e ambientali22, deve proporsi come elemento di riferimento per rendere concreto il principio costituzionale del "diritto al paesaggio". Mi auguro che il convegno "Science and the future" costituisca un passo concreto in questa direzione.

20

E' di questi giorni l'inondazione che ha colpito le Sardegna provocando 17 vittime, ma eventi alluvionali con tragiche conseguenze si registrano sempre più frequentemente in tutto il nostro Paese e sono da mettere in relazione ai cambiamenti climatici, ma soprattutto alla impermeabilizzazione dei suoli, all'edificazione in alvei fluviali e agli scarsi investimenti nella cura e nella messa in sicurezza del territorio. Su questi temi esiste un'ampia letteratura: da ultimo E. Scalfari, Difendere il paesaggio da nuove catastrofi, in "La Repubblica", 22 novembre 2013.

21

Per aggiornamenti e informazioni si veda il Forum "Salviamo il Paesaggio. Difendiamo i territori". www.salviamoilpaseaggio.it

22

E. Dellapiana, G. Montanari, C. Roggero (a cura di), Il patrimonio architettonico e ambientale, Celid, Torino 2007. 